\title{
Recent Progress in the Interpretation of a-Si:H Transport Properties: Lifetimes, Mobilities and Mobility-Lifetime Products
}

\author{
N. Wyrsch ${ }^{1}$, N. Beck ${ }^{1}$, P. Pipoz ${ }^{1}$, M. Goerlitzer ${ }^{1}$, H. Beck ${ }^{2}$ and A. Shah ${ }^{1}$ \\ ${ }^{1}$ Institut de Microtechnique, Breguet 2, $\mathrm{CH}-2000$ Neuchâtel, Switzerland \\ ${ }^{2}$ Institut de Physique, Breguet 1, CH-2000 Neuchâtel, Switzerland
}

Keywords: Transport Properties, Mobility-Lifetime Products, Mobility, Solar Cell, Efficiency

\begin{abstract}
Comparative $\mu \tau$-product measurements performed on a series of slightly doped (i.e. so called "micro-doped") a-Si:H films by steady-state photocarrier grating (SSPG), steady-state photoconductivity (SSPC) and time of flight (TOF) are presented. The observed discrepancy between transient and steady-state majority carriers $\mu \tau$-products (also called the " $\mu \tau$ problem") is discussed, within the framework of a dangling bond recombination model. Using the same model, a material quality related parameter $\mu^{\circ} \tau^{\circ}$ is defined, which allows one to compare and evaluate the quality of layers with different Fermi level positions. Furthermore, correlation between this $\mu^{\circ} \tau^{\circ}$-product, as evaluated for a series of films, grown at different temperatures and the efficiency of solar cells incorporating the corresponding material as an intrinsic layer is demonstrated. Finally, the electron mobility for two series of samples deposited at different temperatures is evaluated by additionnal use of transient photoconductivity decay (TPC) in the small-signal regime.
\end{abstract}

\section{Introduction}

Mobility-lifetime products $\mu \tau$ play a central role in the description of transport properties of devices. However, $\mu \tau$ values deduced experimentally are strongly dependent on the experimental conditions, on the time regime of the experiment (steady-state or transient experiment) and on the Fermi level position in the measured film. In order to compare different experiments or different samples, one has to understand in detail how the measured $\mu \tau$ products depend on all these factors.

In order to compare films deposited under different conditions, it is necessary to rely on a parameter which expresses the "actual" quality of the material and is independent on characteristics like the Fermi level position. Experimental $\mu \tau$ values deduced from SSPC or SSPG are dependent on the occupation of the dangling bonds and, on the other hand, the measured defect density alone does not fully reflect actual transport quality. Consequently, all these parameters are not suitable as a quality monitor, e.g. for solar cells. The major aim of this paper is, thus, to introduce a parameter which satisfies all the above conditions.

As far as modelisation of transport in films or solar cells is concerned, it is often necessary not only to evaluate the $\mu \tau$-product, but also to separate mobilities and lifetimes. A procedure is therefore also needed to measure independently $\mu$ or $\tau$. By combining both SSPC results and lifetime measurements by TPC, one can successfully evaluate $\mu$.

\section{Theory: $\mu \tau$ products}

Transport properties of a material such as a-Si:H is generally described in terms of mobility-lifetime products ( $\mu \tau$ products), for both electrons and holes. However, the $\mu \tau$ values obtained experimentally reflect the situation in the material (given by all the trapping, reemission and recombination events) under the prevailing experimental conditions. They, thus, generally depend strongly on the occupation of the dangling bonds. Let us first briefly discuss $\mu \tau$ values obtained from different measurement techniques within the framework of a standard recombination model via dangling bond states (see Fig. 1).

Time of Flight (TOF). In the (transient) TOF experiment, the observed mechanism is multiple trapping for the considered carrier (and reemission by tail states) and its final capture on a dangling bond state $[1,2]$. Only one type of carrier is involved, and thermal emission from the dangling bond 


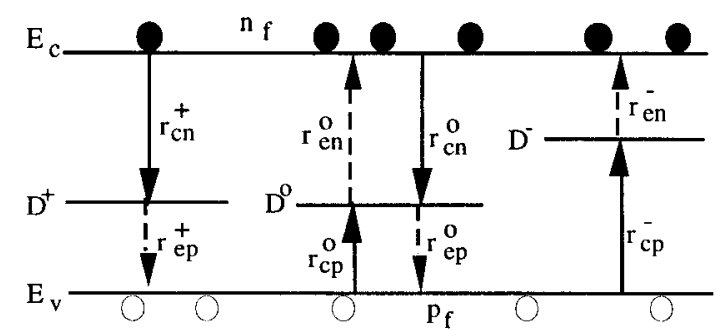

Fig. 1: Allowed transitions in the dangling bond recombination model, according to [4].

states can be excluded, by an appropriate choice of the time window for the experiment. The $\mu \tau$ product is here a drift mobility $x$ deep trapping time $\mu^{\mathrm{D}} \tau^{\mathrm{D}}$ product which can be easily identified with a band mobility $x$ free carrier deep-trapping time product $\mu^{\circ} \tau$ [3]. The free carrier deep trapping time for electrons $\tau_{\mathrm{n}}$ and for holes $\tau_{\mathrm{p}}$ can be written as:

$$
\tau_{\mathrm{n}}=\frac{1}{\mathrm{v}_{\mathrm{th}} \mathrm{N}_{\mathrm{db}} \sigma_{\mathrm{n}}^{\mathrm{o}} \mathrm{f}_{\mathrm{o}}^{\mathrm{o}}+\mathrm{v}_{\mathrm{th}} \mathrm{N}_{\mathrm{db}} \sigma_{\mathrm{n}}^{+} \mathrm{f}_{\mathrm{o}}^{+}}, \tau_{\mathrm{p}}=\frac{1}{\mathrm{v}_{\mathrm{th}} \mathrm{N}_{\mathrm{db}} \sigma_{\mathrm{p}}^{\mathrm{o}} \mathrm{f}_{\mathrm{o}}^{\mathrm{o}}+\mathrm{v}_{\mathrm{th}} \mathrm{N}_{\mathrm{db}} \sigma_{\mathrm{p}}^{-} \mathrm{f}_{\mathrm{o}}^{-}},
$$

where $\mathrm{f}_{\mathrm{o}}^{\mathrm{o}}, \mathrm{f}_{\mathrm{o}}^{+}, \mathrm{f}_{\mathrm{o}}^{-}$are the occupation functions of the dangling bonds at thermal equilibrium (TOF experiments are performed in the dark), $v_{t h}$ is the thermal velocity $\left(v_{t h}\right.$ is assumed, for the sake of simplicity, to be the same for both electrons and holes; nevertheless, a different assumption would not modified significantly the following development), $N_{d b}$ is the deep defect density and $\sigma_{n}^{o}, \sigma_{n}^{+}$, $\sigma_{\mathrm{p}}^{\mathrm{o}}, \sigma_{\mathrm{p}}^{-}$are the capture cross-sections of the dangling bonds. As a consequence, $\tau_{\mathrm{n}}$ and $\tau_{\mathrm{p}}$ depend strongly on the occupation functions which are given by the dark Fermi level $\mathrm{E}_{\mathrm{F}}^{\mathrm{O}}$, and on the correlation energy $U$ [5] (one should note that this dependency is especially pronounced when $E_{F}^{0}$ shifts around midgap and provided one assumes $\left.\sigma_{n}^{+} / \sigma_{n}^{o}=\sigma_{p}^{-} / \sigma_{p}^{o} \gg 1\right)$.

Photoconductivity (SSPC) and Steady-State Photocarrier Grating (SSPG). In these steadystate measurement methods, an equilibrium is achieved between generation and recombination. The transport mechanism is described in terms of band mobility $x$ free carrier recombination time $\mu^{o} \tau^{R}$, where the characteristic times $\tau_{\mathrm{n}}^{\mathrm{R}}$ and $\tau_{\mathrm{p}}^{\mathrm{R}}$ are given by:

$$
\mathrm{G}=\mathrm{R}=\frac{\mathrm{n}_{\mathrm{f}}}{\tau_{\mathrm{n}}^{\mathrm{R}}}=\frac{\mathrm{p}_{\mathrm{f}}}{\tau_{\mathrm{p}}^{\mathrm{R}}},
$$

where $G$ is the generation rate, $R$ is the recombination rate and $n_{f}, p_{f}$ are the free electron and the free hole density, respectively. The photoconductivity $\sigma_{\mathrm{ph}}$ as obtained from SSPC is then given by:

$$
\sigma_{\mathrm{ph}}=\mathrm{e}\left(\mu_{\mathrm{n}}^{\mathrm{o}} \mathrm{n}_{\mathrm{f}}+\mu_{\mathrm{p}}^{\mathrm{o}} \mathrm{p}_{\mathrm{f}}\right)=\mathrm{eG}\left(\mu_{\mathrm{n}}^{\mathrm{o}} \tau_{\mathrm{n}}^{\mathrm{R}}+\mu_{\mathrm{p}}^{\mathrm{o}} \tau_{\mathrm{p}}^{\mathrm{R}}\right) \text {, }
$$

where $e$ is the elementary charge. The ambipolar diffusion length $L_{a m b}$ deduced from SSPG can be written as:

$$
\mathrm{L}_{\mathrm{amb}}^{2}=\frac{\mathrm{kT}}{\mathrm{e}} \frac{\mu_{\mathrm{n}}^{\mathrm{o}} \tau_{\mathrm{n}}^{\mathrm{R}} \mu_{\mathrm{p}}^{\mathrm{o}} \tau_{\mathrm{p}}^{\mathrm{R}}}{\mu_{\mathrm{n}}^{\mathrm{o}} \tau_{\mathrm{n}}^{\mathrm{R}}+\mu_{\mathrm{p}}^{\mathrm{o}} \tau_{\mathrm{p}}^{\mathrm{R}}} \mathrm{C},
$$

where $\mathrm{kT}$ is the thermal energy and $\mathrm{C}$ a correction factor evaluated to be between 1 and 2 [6]. One should note that, when the material has a clear n- or p-type character, equations (3) and (4) can be greatly simplified by neglecting the minority carrier contribution in $\sigma_{\mathrm{ph}}$ and the majority carrier contribution in $\mathrm{L}_{\mathrm{amb}}$. The position of the Fermi level $\mathrm{E}_{\mathrm{F}}$ can be expressed by the parameter $\mathrm{b}$ which is defined [7], as:

$$
\mathrm{b}=\frac{\mu_{\mathrm{r}}^{o} \mathrm{n}_{\mathrm{f}}}{\mu_{\mathrm{p}}^{\mathrm{o}} \mathrm{p}_{\mathrm{f}}}=\frac{\mu_{\mathrm{n}}^{\mathrm{o}} \tau_{\mathrm{n}}^{\mathrm{R}}}{\mu_{\mathrm{p}}^{\mathrm{o}} \tau_{\mathrm{p}}^{\mathrm{R}}} .
$$

The parameter $\mathbf{b}$ can be experimentally evaluated from the following expression (which can be deduced from equations (3) and (4)):

$$
\frac{b}{(b+1)^{2}}=\frac{L_{a m b}^{2} e^{2} G}{k T \sigma_{p h} C} \text {. }
$$

The recombination times $\tau_{n}^{\mathrm{R}}$ and $\tau_{p}^{\mathrm{R}}$ (involved in equations (2-5)) take into account all recombination processes via all available recombination centres. In the dangling bond recombination model given in Fig. 1, under sufficiently high illumination (corresponding to the usual conditions for SSPC and SSPG measurement), quasi Fermi levels for trapped electrons and for trapped holes are 
pushed outside of the dangling bond distribution and, thus, the latter can be identified with the recombination centre distribution (as long as the quasi Fermi levels are not pushed too far into the band tails). All reemission from the dangling bond states can then be neglected (dotted arrows in Fig. 1) and the recombination times can be written as:

$$
\tau_{\mathrm{n}}^{\mathrm{R}}=\frac{1}{\mathrm{v}_{\mathrm{th}} \mathrm{N}_{\mathrm{db}} \sigma_{\mathrm{n}}^{\mathrm{o}} \mathrm{f}^{\mathrm{o}}+\mathrm{v}_{\mathrm{th}} \mathrm{N}_{\mathrm{db}} \sigma_{\mathrm{n}}^{+} \mathrm{f}^{+}}, \tau_{\mathrm{p}}^{\mathrm{R}}=\frac{1}{\mathrm{v}_{\mathrm{th}} \mathrm{N}_{\mathrm{db}} \sigma_{\mathrm{p}}^{0} \mathrm{f}^{\mathrm{o}}+\mathrm{v}_{\mathrm{th}} \mathrm{N}_{\mathrm{db}} \sigma_{\mathrm{p}}^{-} \mathrm{f}^{-}},
$$

where $\mathrm{f}^{0}, \mathrm{f}^{+}, \mathrm{f}$ are the dangling bond occupation functions in steady-state equilibrium (under illumination). These occupation functions can be expressed analytically in terms of the free electron and free hole densities and their capture times on the specific dangling bond states $[4,5]$ :

$$
\begin{aligned}
& \mathrm{f}^{\mathrm{o}}=1 /\left(\frac{\mathrm{p}_{\mathrm{f}}}{\mathrm{n}_{\mathrm{f}}} \frac{\sigma_{\mathrm{p}}^{\mathrm{o}}}{\sigma_{\mathrm{n}}^{+}}+1+\frac{\mathrm{n}_{\mathrm{f}}}{\mathrm{p}_{\mathrm{f}}} \frac{\sigma_{\mathrm{n}}^{\mathrm{o}}}{\sigma_{\mathrm{p}}^{-}}\right), \\
& \mathrm{f}^{-}=\frac{\mathrm{n}_{\mathrm{f}}}{\mathrm{p}_{\mathrm{f}}} \frac{\sigma_{\mathrm{n}}^{\mathrm{o}}}{\sigma_{\mathrm{p}}^{-}} /\left(\frac{\mathrm{p}_{\mathrm{f}}}{\mathrm{n}_{\mathrm{f}}} \frac{\sigma_{\mathrm{p}}^{o}}{\sigma_{\mathrm{n}}^{+}}+1+\frac{\mathrm{n}_{\mathrm{f}}}{\mathrm{p}_{\mathrm{f}}} \frac{\sigma_{\mathrm{n}}^{\mathrm{o}}}{\sigma_{\mathrm{p}}^{-}}\right), \\
& \mathrm{f}^{+}=\frac{\mathrm{p}_{\mathrm{f}}}{\mathrm{n}_{\mathrm{f}}} \frac{\sigma_{\mathrm{p}}^{\mathrm{o}}}{\sigma_{\mathrm{n}}^{+}} /\left(\frac{\mathrm{p}_{\mathrm{f}}}{\mathrm{n}_{\mathrm{f}}} \frac{\sigma_{\mathrm{p}}^{\mathrm{o}}}{\sigma_{\mathrm{n}}^{+}}+1+\frac{\mathrm{n}_{\mathrm{f}}}{\mathrm{p}_{\mathrm{f}}} \frac{\sigma_{\mathrm{n}}^{\mathrm{o}}}{\sigma_{\mathrm{p}}^{-}}\right) .
\end{aligned}
$$

$\mathrm{f}^{0}, \mathrm{f}^{+}, \mathrm{f}^{-}$are independent of the energy distribution (as long as the capture cross-sections are not a function of energy) and of the correlation energy of the recombination centres. Note that, in this particular situation, characteristic times $\tau_{n}, \tau_{p}$ for TOF and $\tau_{n}^{R}, \tau_{p}^{R}$ for SSPC and SSPG become formally identical.

Definition of $\mu^{\circ} \tau^{\circ}$ products. As all $\mu \tau$ products presented above are function of the dangling bond occupation, we now define transport parameters $\mu^{\circ} \tau^{\circ}$ which are "true" material parameter and do not depend on the dangling bond charge:

$$
\mu_{\mathrm{n}}^{\mathrm{o}} \tau_{\mathrm{n}}^{\mathrm{o}}=\mu_{\mathrm{n}}^{\mathrm{o}} \frac{1}{\mathrm{v}_{\mathrm{th}} \mathrm{N}_{\mathrm{db}} \sigma_{\mathrm{n}}^{\mathrm{o}}}, \mu_{\mathrm{p}}^{\mathrm{o}} \tau_{\mathrm{p}}^{\mathrm{o}}=\mu_{\mathrm{p}}^{\mathrm{o}} \frac{1}{\mathrm{v}_{\mathrm{th}} \mathrm{N}_{\mathrm{db}} \sigma_{\mathrm{p}}^{\mathrm{o}}}
$$

In general, these parameters cannot be directly measured since they are linked to the situation where all dangling bonds are in the $\mathrm{D}^{\circ}$ state. However, they are indeed material characteristics which describe the quality of the material in terms of band mobilities and defect density. At this point, we can introduce the following simplification:

$$
\mu_{\mathrm{n}}^{\mathrm{o}} \tau_{\mathrm{n}}^{\mathrm{o}}=\mu_{\mathrm{p}}^{\mathrm{o}} \tau_{\mathrm{p}}^{\mathrm{o}}=\mu^{\mathrm{o}} \tau^{\mathrm{o}}
$$

this equality is based on previous TOF measurements performed by our group on slightly doped and compensated sample in various degradation states $[8,9]$. These measurements show (see Fig. 2), for strongly degraded samples, where almost all dangling bonds are neutral, that $\mu_{n}^{\mathrm{o}} \tau_{\mathrm{n}}^{\mathrm{o}}$ and $\mu_{\mathrm{p}}^{\mathrm{o}} \tau_{\mathrm{p}}^{\mathrm{o}}$ are, in fact, almost equal $\left(\mu_{\mathrm{n}}^{\mathrm{o}} \tau_{\mathrm{n}}^{\mathrm{o}} / \mu_{\mathrm{p}}^{\mathrm{o}} \tau_{\mathrm{p}}^{\mathrm{o}} \approx 1.2\right)$.

${ }_{\mathrm{p}} \tau_{\mathrm{p}}$ are, in fact, almost equation of $\mu^{\mathbf{0}} \tau^{\mathbf{0}}$ products. The recombination times $\tau_{\mathrm{n}}^{\mathrm{R}}$ and $\tau_{\mathrm{p}}^{\mathrm{R}}$ are not independent of each

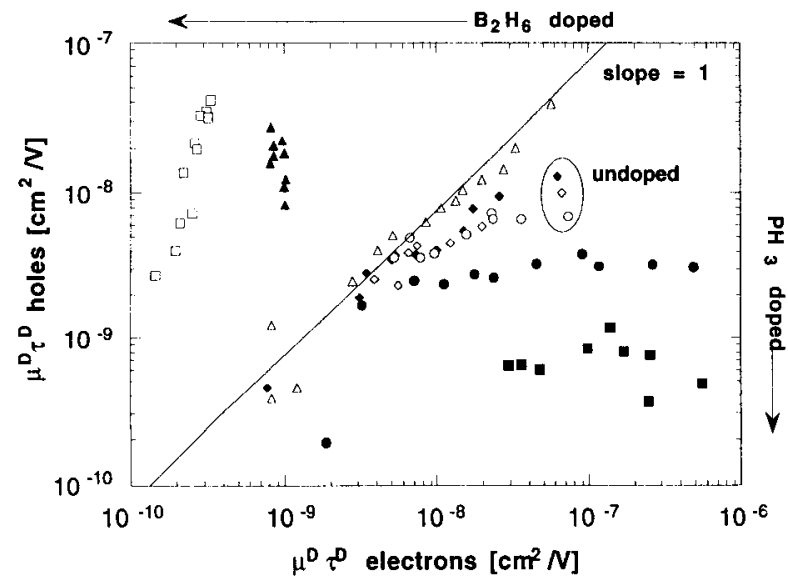

Fig. 2: Electron versus hole $\mu^{D} \tau^{D}$ products in the annealed state and their change induced by lightsoaking. The line with slope 1 indicates the ratio of hole $\mu_{p} \tau_{p}$ to electron $\mu_{n}{ }^{o} \tau_{n}$ for compensated ("midgap") or strongly degraded samples. 
other, but exhibit an anticorrelated behaviour as a function of the Fermi level position, or, alternatively expressed, as a function of the parameter $b[8,10,11]$, since majority and minority carriers properties are coupled via the same recombination centers (the dangling bonds). We can, thus, take advantage of this fact and determine $\mu^{\circ} \tau^{\circ}$-product that is independent of a dangling bond charge. By introducing the functions (8-10) into the expressions (7), and taking into account the hypothesis (12), we obtain the following results:

$$
\mu_{\mathrm{n}}^{\mathrm{o}} \tau_{\mathrm{n}}^{\mathrm{R}}=\mu^{\mathrm{o}} \tau^{\mathrm{o}} \frac{\mathrm{b}}{\mathrm{b}+1} \mathrm{z}, \mu_{\mathrm{p}}^{\mathrm{o}} \tau_{\mathrm{p}}^{\mathrm{R}}=\mu^{\mathrm{o}} \tau^{\mathrm{o}} \frac{1}{\mathrm{~b}+1} \mathrm{z},
$$

with

$$
\mathrm{z}=\frac{1}{\mathrm{f}^{\mathrm{o}}}=\left(\frac{\sigma_{\mathrm{n}}^{\mathrm{o}}}{\sigma_{\mathrm{n}}^{+}} \frac{1}{\mathrm{~b}}+1+\frac{\sigma_{\mathrm{p}}^{\mathrm{o}}}{\sigma_{\mathrm{p}}^{-}} \mathrm{b}\right) .
$$

The expressions (13) show that the values for $\mu_{n}^{o} \tau_{n}^{R}$ and $\mu_{p}^{o} \tau_{p}^{R}$, as deduced experimentally from SSPC and SSPG, need to be corrected by a factor which is a function of the parameter $b$ (i.e. a function of Fermi level position, and, thus a function of both intentional and non-intentional doping). Nevertheless, for "truly intrinsic" (compensated or "midgap") material, both majority and minority carriers contribute to $\sigma_{p h}$ and $L_{a m b}$ and the value of $\mu_{n}^{\mathrm{o}} \tau_{n}^{\mathrm{R}}$ and $\mu_{\mathrm{p}}^{\mathrm{o}} \tau_{\mathrm{p}}^{\mathrm{R}}$ cannot be evaluated. Because of this, it is convenient to replace the expressions (13) in the expression of the photoconductivity (3) and we obtain:

$$
\mu^{\mathrm{o}} \tau^{\mathrm{o}}=\frac{\sigma_{\mathrm{ph}}}{\mathrm{eG}} \cdot \frac{1}{\mathrm{z}}
$$

This compact formula is now valid for all types of a-Si:H; the character of the material, monitored by the parameter $b$, which influences our correction factor $z$. Note that measurements of $\mathrm{L}_{\mathrm{amb}}$ only appear through the parameter $b$. The magnitude of the correction factor $z$, as a function of $b$ (for given $\sigma_{\mathrm{n}}^{+} / \sigma_{\mathrm{n}}^{0}$ and $\sigma_{\mathrm{p}}^{-} / \sigma_{\mathrm{p}}^{0}$ ) is shown in Fig. 3 .

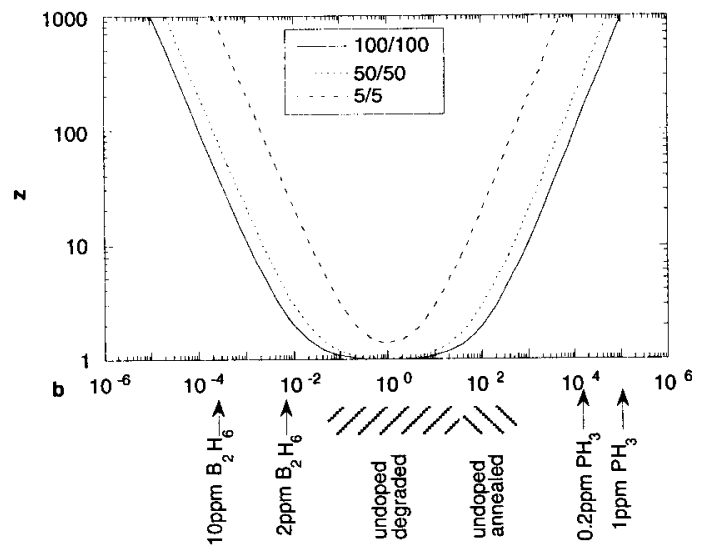

Fig. 3: Calculated value of the correction factor for experimental $\mu^{\theta} \tau^{R}$-products, as deduced from photoconductivity (see below), for different of the parameter $b$. The correction factor $z$ is needed in order to obtain quality-related $\mu^{0} \tau^{0}$-products. The inboxd indicates the different ratios of capture cross-sections $\sigma_{n}^{+} / \sigma_{n}{ }^{\circ}$ and $\sigma_{p}{ }^{-} / \sigma_{p}^{o}$ used in the calculation. Along the horizontal axis some typical values for the parameter $b$, as observed on undoped (annealed and degraded) and slightly doped (only annealed) a-Si:H layers, are indicated.

Lets us now consider some particular cases:

For truly intrinsic material (where the Fermi level is near midgap), $b \approx 1$ and the correction factor is close to 1 . Thus, $\mu_{n}^{\mathrm{O}} \tau_{\mathrm{n}}^{\mathrm{R}}$ (deduced from $\sigma_{\mathrm{ph}}$ ) and $\mu^{\circ} \tau^{\mathrm{O}}$ become identical; $\sigma_{\mathrm{ph}}$ is here the proper parameter to describe material quality.

For n-type material (or even for undoped slightly n-type films), as soon as $b>\sigma_{p}^{-} / \sigma_{p}^{o}$, and for p-type material, as soon as $b<\sigma_{n}^{\circ} / \sigma_{n}^{+}$, the material quality parameter $\mu^{\mathrm{o}} \tau^{\mathrm{o}}$ is given by $\mathrm{L}_{\mathrm{anb}}$ and:

$$
\mu^{\mathrm{o}} \tau^{\mathrm{o}} \approx \mathrm{L}_{\text {amb }}^{2} \frac{1}{\mathrm{C}} \frac{\mathrm{e}}{\mathrm{kT}} \frac{\sigma^{ \pm}}{\sigma^{\mathrm{o}}} \text {. }
$$

As mentioned above, the constant $\mathrm{C}$ has an approximate value of 1.5. Unfortunately, values of the capture cross-section ratios $\sigma_{n}^{+} / \sigma_{n}^{\circ}$ and $\sigma_{p}^{-} / \sigma_{p}^{0}$ are very controversial; values found in the literature vary between $1.5[12]$ and $100[9,13]$. However, as will be shown below (see Fig. 6), the dependence $\mu^{\circ} \tau^{\circ}$ which was measured by us as a function of the doping, suggests a value of $\sigma^{2} / \sigma^{\circ}=50$, which is consistent with the value obtained from previous TOF studies [8,9]. Therefore, this latter value will be used for the present study. 


\section{Experimental}

All samples used in this study were deposited by the VHF glow discharge deposition technique (at $\mathrm{f}=70 \mathrm{MHz}$ ) [14]. Deposition temperature was $220^{\circ} \mathrm{C}$, unless otherwise indicated. For steadystate experiments, the a-Si:H layers were deposited on glass substrates (Corning 7059), whereas for the TOF samples, the glass substrate was $\mathrm{Cr}$-coated before deposition. TOF and steady-state samples were produced in the same deposition run. The thicknesses of the films varied between $2.2 \mu \mathrm{m}$ and $5 \mu \mathrm{m}$ for the study on the $\mu \tau$ problem, between 2.2 and $3.8 \mu \mathrm{m}$ for the study on the correlation between layers and cells and, finally, between 1.8 and $2.6 \mu \mathrm{m}$ for the mobility study. For micro-doped samples, the volumic gas concentration varied between $0.2 \mathrm{ppm}$ and $1 \mathrm{ppm}$ of $\mathrm{PH}_{3}$ and 0.5 and $10 \mathrm{ppm}$ of $\mathrm{B}_{2} \mathrm{H}_{6}$ in pure silane gas. For the steady-state and TPC experiments, two coplanar aluminium contacts separated by a gap of $0.5 \mathrm{~mm}$, were evaporated on the films. For TOF samples, a semitransparent $\mathrm{Cr}$ front-contact was evaporated to form a double Schottky diode sandwich structure $(\mathrm{Cr}(1500 \AA) / \mathrm{a}-\mathrm{Si}: \mathrm{H} / \mathrm{Cr}(150 \AA))$.

All samples were annealed at $180^{\circ} \mathrm{C}$ for 2 hours prior to their characterisation. To indicate the doping level, we subsequently used the parameter $b$ as introduced above. For SSPC and SSPG, a Krypton laser $\left(\lambda=647 \mathrm{~nm}, 3\right.$ to $\left.90 \mathrm{~mW} / \mathrm{cm}^{2}\right)$ was used, allowing generation of electron hole pairs with a rate of $2-60 \times 10^{19} \mathrm{~cm}^{-3} \mathrm{~s}^{-1}$ in the film.

For TPC experiments, illumination was obtained from a HeNe laser at $633 \mathrm{~nm}(15 \mathrm{~mW})$. The bias light intensity was adjusted by using neutral density filters. The beam was then focused through an acousto-optic modulator with a rise time of $60 \mathrm{~ns}$, in order to provide for small steps in the illumination (5-10\% of the bias light level). Transient photocurrent was detected using a probe amplifier and recorded on a digitizing oscilloscope. Data was then transferred on a computer for processing.

\section{The $\mu \tau$-problem}

The discrepancy between majority carrier $\mu \tau$-products measured by steady-state experiments (SSPC or SSPG) and transient experiments (TOF) has in the past been called the " $\mu \tau$-problem" [3]. As shown in Fig. 4, majority carrier $\mu \tau$ may differ by up to 2 orders of magnitude, whereas minority carrier $\mu \tau$ are comparable. Several attempts at explaining this discrepancy have been made, but so far they are all quite unsuccessful. Isotropy of the transport properties (SSPC and SSPG measurements are performed in coplanar configuration, whereas TOF measurement are performed in sandwich configuration) has been confirmed for a-Si:H, at least for our "standard" VHF-GD samples [3], and thus cannot account for this discrepancy.

Some authors have suggested that thermal emission is the key explanation $[3,15,16]$. Photogenerated carriers may undergo several trapping events on a deep trap and several thermal reemission events, before they finally recombine. As a consequence, $\mu \tau$-values measured in steadystate, involving recombination lifetimes, should be larger than $\mu \tau$-values measured in TOF, since, in the latter, only the first trapping event is recorded (reemission is forbidden by the choice of a short experimental time window); in other words, the recombination time should be (according to
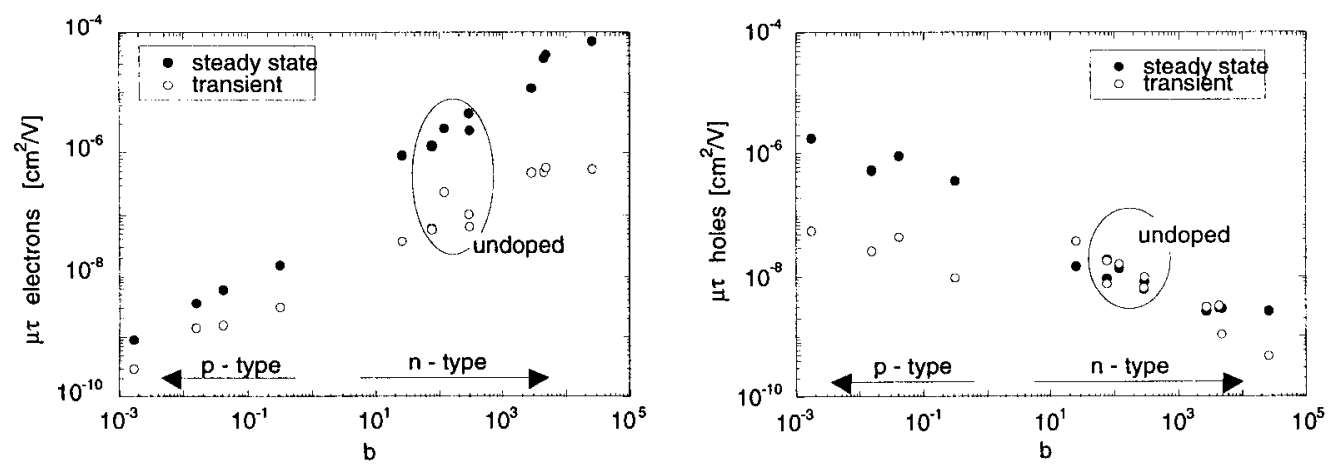

Fig. 4: Steady-state and transient $\mu \tau$-products as a function of the doping level, the latter being monitored by the parameter $b$ (see text). 
this reasoning) much longer than the trapping time.

This reasoning certainly holds in the dark, where thermal equilibrium requires the trapping rate to be equal to the emission rate. The latter can thus be estimated from the dark conductivity and $\mu^{\mathrm{D}} \tau^{\mathrm{D}}$ measured by TOF. For $\mathrm{n}$-type samples, where most of the dangling bonds are negatively charged, $\mu_{n}^{D} \tau_{n}^{D}$ will be (according to (1) and Fig. 1):

$$
\mu_{\mathrm{n}}^{\mathrm{D}} \tau_{\mathrm{n}}^{\mathrm{D}}=\mu_{\mathrm{n}}^{\mathrm{o}} \tau_{\mathrm{n}} \approx \frac{\mu_{\mathrm{n}}^{o}}{\mathrm{v}_{\mathrm{th}} N_{\mathrm{db}} \sigma_{\mathrm{n}}^{\mathrm{o}} \mathrm{f}_{\mathrm{o}}^{\mathrm{o}}}=\mu_{\mathrm{n}}^{\mathrm{o}} \frac{\mathrm{n}_{\mathrm{f}}}{\mathrm{r}_{\mathrm{cn}}^{\mathrm{o}}}=\mu_{\mathrm{n}}^{\mathrm{o}} \frac{\mathrm{n}_{\mathrm{f}}}{\mathrm{r}_{\mathrm{en}}^{-}} \text {. }
$$

Using the expressions (18) and (2) for $\mathrm{n}_{\mathrm{f}}$ in the dark, we obtain

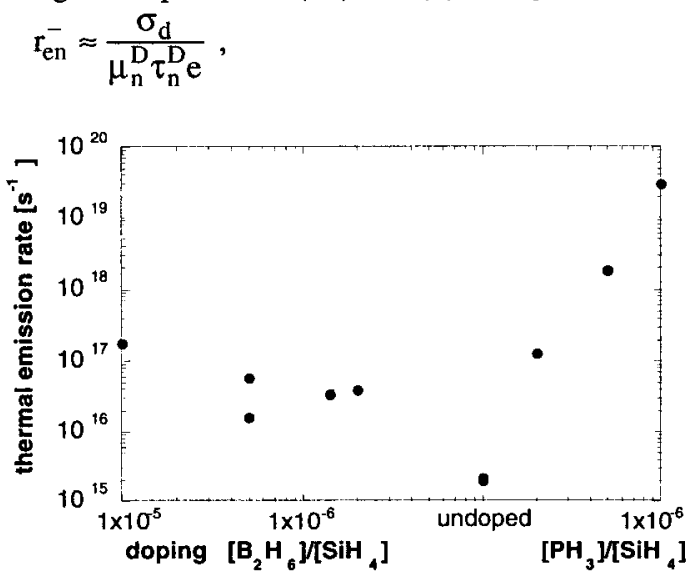

which is an estimate of the thermal emission. After a similar calculation for p-type samples, we can deduce the thermal emission data as given in Fig. 5. Thermal emission rates are to be calculated at thermal equilibrium, in the dark, and are therefore not a function of illumination. The generation rates of the steady-state measurements carried out by us (SSPC or SSPG), are above $10^{19} \mathrm{~cm}^{-3} \mathrm{~s}^{-1}$, and are cartainly much larger than the estimated thermal emission rates (Fig. 5). At these generation levels, quasi Fermi levels for trapped carriers lay outside of the dangling bond distribution, and thus, the latter do not play the role of traps. Consequently, thermal emission cannot be used as an explanation for the $\mu \tau$-problem (at least for undoped or slightly doped samples).

Comparing the formal expressions (1) and (7) of the $\mu \tau$ product for TOF, and for SSPC/SSPG, respectively, we observe that the only difference lies in the occupation functions (dark occupation functions for TOF and occupation pertaining to steady-state illumination for SSPC and SSPG). Indeed, simple calculations within the framework of the present recombination model lead to a qualitative explanation for the discrepancy known as the " $\mu \tau$-problem" [9]. However, as the occupation functions depend critically on the distribution of the defects, on their correlation energy and on the generation rate, a precise quantitative "solution" of the $\mu \tau$-problem remains problematic.

\section{Transport properties and micro-doping}

Small changes in the Fermi level (e.g. by doping, even unintentional) have a dramatic effect on the transport properties $[8,9,17]$. Due to the anticorrelated behaviour of $\mu \tau$-products for minority and majority carriers, an individual measurement of SSPC or SSPG will be unable to give a direct indication for the quality of the material; an evaluation of the product $\mu^{\circ} \tau^{\circ}$ is required. In Fig. 6 , experimental results for $\mu^{0} \tau^{R}$, and for the resulting product $\mu^{0} \tau^{0}$, evaluated according to the procedure described above, are presented. We can observe that the $\mu^{0} \tau^{0}$ values, which are proportional to $1 / \mathrm{N}_{\mathrm{db}}$, follow the $1 / \sqrt{[\text { dopant }] /\left[\mathrm{SiH}_{4}\right]}$ law (as indicated by a dotted line) [17] provided a ratio of capture cross-section $\sigma^{ \pm} / \sigma^{0}=50$ is chosen (note that the square root law is always observed, for n-type material, as long as $\sigma^{ \pm} / \sigma^{0}<2000$ ). 


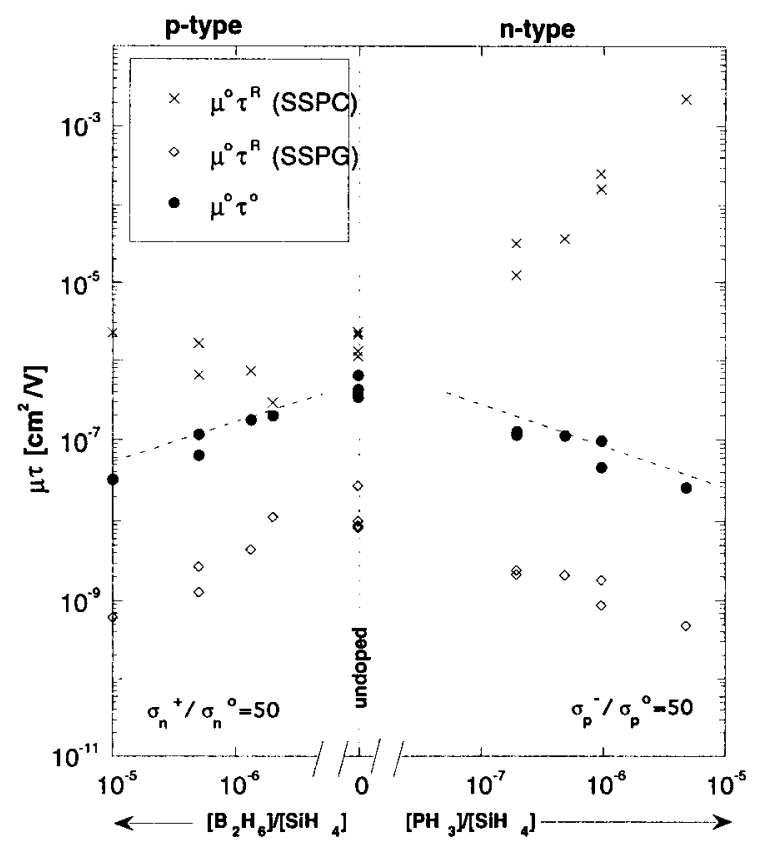

Fig. 6: variation in the measured products $\mu_{n}^{o} \tau_{n}^{R}, \quad \mu_{p}^{o} \tau_{p}^{R}$ and in the product $\mu^{0} \tau^{\partial}$ deduced from these measurements as a function of low level $\mathrm{PH}_{3}$ and $\mathrm{B}_{2} \mathrm{H}_{6}$ doping. A ratio of capture cross sections $\sigma_{n}{ }^{+} / \sigma_{n}{ }^{\circ}$ and $\sigma_{p}{ }^{-} / \sigma_{p}{ }^{\circ}$ of 50 was chosen for the plot (see text). The $1 / \sqrt{\left[\mathrm{B}_{2} \mathrm{H}_{6}\right] /\left[\mathrm{SiH}_{4}\right]}$ law for defect densities is represented by the dotted lines.

\section{Transport properties and solar cell performance}

The quality-related product $\mu^{\circ} \tau^{\circ}$ was mainly introduced by us with the aim to correlate material transport properties and solar cell performances. In order to check the validity of the method, two series of films and cells (the latter incorporating the same material as an i-layer) were deposited by VHF-GD, at deposition temperatures between 120 and $320^{\circ} \mathrm{C}$. The degraded state (for the first series only) was obtained, for the cells, after 3 weeks of illumination by an AM1.5 light source at $100 \mathrm{~mW} \mathrm{~cm}^{-2}$ (array of PL-L 24W/95/4P fluorescent tubes), and for the layers, after 4 weeks of illumination by a 6 sun high pressure sodium lamp (with a spectral maximum at $590 \mathrm{~nm}$ ).

Correlation between the $\mu^{\circ} \tau^{\circ}$ value of the film and the cell efficiency, in the annealed and the degraded state, is shown in Fig. 7. A very good correlation is obtained for both annealed and degraded states. Material quality increases with temperature and this is indeed reflected in the

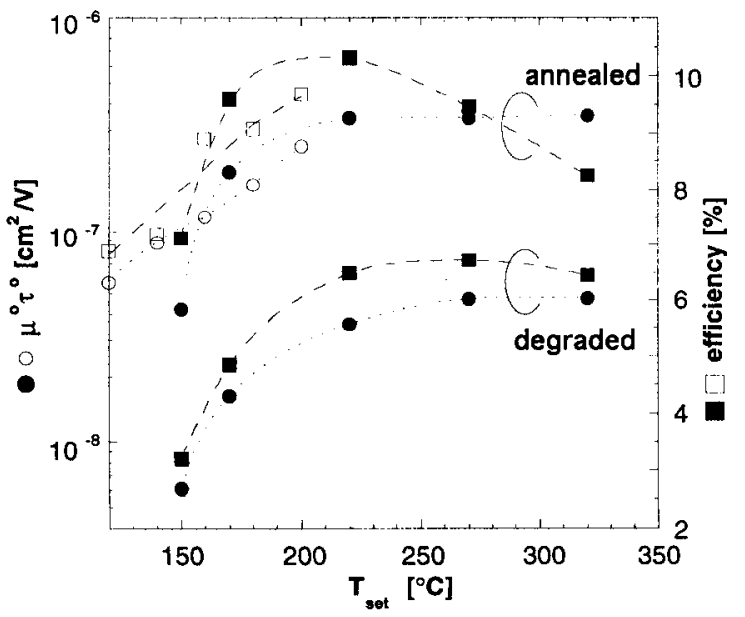

Fig. 7: $\mu^{o} \tau^{\prime}$ products evaluated on two series of $2.5 \mu \mathrm{m}$ thick, undoped films and corresponding efficiencies of solar cells incorporating the "same" films as an i-layer, in the annealed state and in the degraded state (first series is plotted with solid marks, second series is plotted with open marks). $T_{\text {set }}$ is the setpoint of the deposition temperature. (For our evaluation of $\mu^{o} \tau$, we assumed $\left.\sigma_{n}^{+} / \sigma_{n}{ }^{\circ}=\sigma_{p}{ }^{-} / \sigma_{p}{ }^{o}=50\right)$. 
increase of the cell efficiency. Above $220^{\circ} \mathrm{C}$, we observe a decrease of the cell efficiency, whereas the $\mu^{\circ} \tau^{\circ}$ values saturate. This discrepancy should (in our opinion) be related here to interdiffusion or interface degradation of the cells at these relatively high temperatures (note that the $V_{o c}$ of these cells also is lower) and not to a failure of the method.

As far as the determination of $\mu^{\circ} \tau^{\circ}$ is concerned, Fig. 8 shows that in the annealed state most of the layers exhibit an extrinsic (n-type) character, and thus, the quality of the film is determined here by $L_{\text {amb }}$. With light soaking, the Fermi level is pushed towards midgap and the films become more intrinsic; $\sigma_{\mathrm{ph}}$ is then the right parameter to evaluate the quality of the layers.

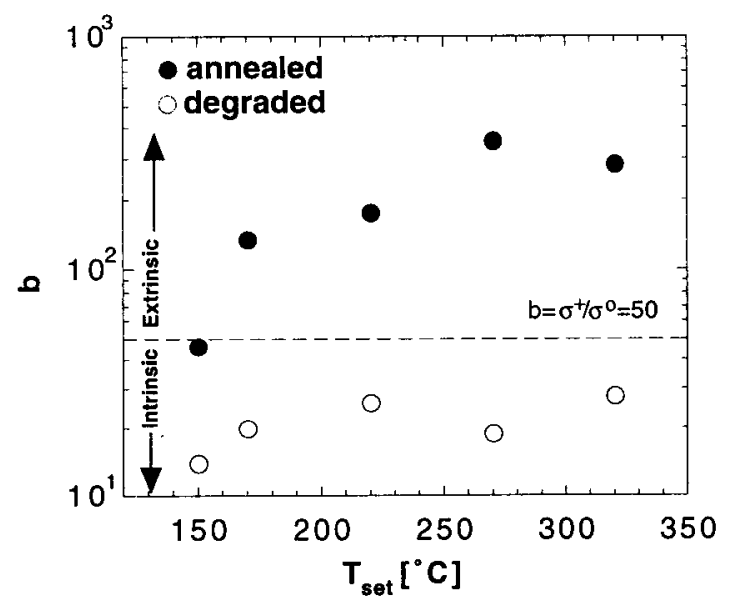

Fig. 8: Variation in the values of the parameter $b$, with degradation, for $a$ temperature-series of undoped layers; while in the annealed state almost all layers have a slightly extrinsic character, they all become intrinsic with lightsoaking. The limit is given by the ratio of capture cross-sections $\sigma^{ \pm} / \sigma^{\circ}$, which was assumed here to be 50 .

\section{Lifetime measurement and microscopic mobilities}

We have developed a theoretical model to describe transients of photoconductivity in the case of small changes of the light excitation [18]. This model, based on the recombination model described above (recombination through capture of free carriers on the dangling bonds, capture and recombination events in band tails) neglects thermal emission from the dangling bonds (as already justified above, during the discussion of the $\mu \tau$-problem), as well as transition from one band to the tail of the other.

From the multiple-trapping equations, analytical solutions for normalized transient photoconductivity are found for certain special cases of the localized state distribution: the bandtail is considered exponential with a slope characteristic parameter $\alpha=T / T_{c}=1$ or $\alpha=0.5$ (these values correspond at $300 \mathrm{~K}$ to a characteristic energy of $25 \mathrm{meV}$, or to $50 \mathrm{meV}$, respectively), the dangling bond distribution is considered a Dirac function (the shape of this distribution does not, in fact, play a role in this model, as long as only one type of recombination centers is considered and reemission

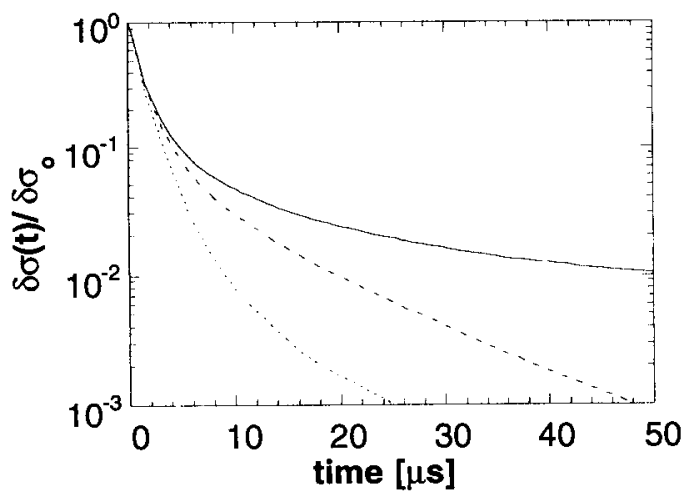

Fig. 9: Theoretical curves for the variation in excess photoconductivity versus time (electron contribution): (a) taking into account an exponential bandtail and the dangling bond distribution (dotted line), (b) taking into account an exponential bandtail the dangling bond and a constant localized state distribution (dashed line), (c) same as case (b) but for both electron and hole contributions (continuous line). 
from the dangling bonds is neglected); furthermore, there is an additional constant localized state distribution (constant with respect to energy, between the bandedge and the quasi Fermi level). Three theoretical curves for specific cases are plotted in Fig. 9. Note that contribution of holes could be neglected for all samples measured in the present study. By fitting of the experimental curves, values for the recombination lifetime $\tau^{\mathrm{R}}$ could be evaluated. Fig. 10 shows experimental curves obtained at different light generation rates. A good agreement between the theory and the measured curves is obtained for a fairly large range of generation rates.

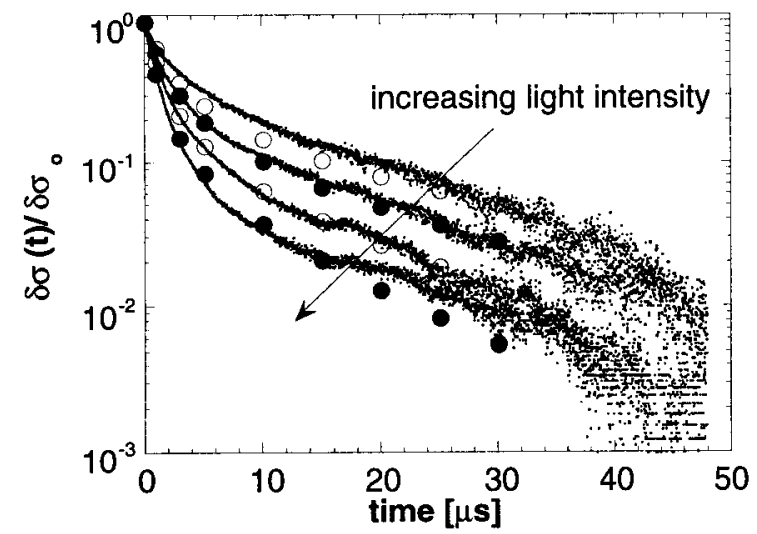

Fig. 10: measured curves for four different bias light intensities (1$20 \mathrm{mWcm}^{-2}$ ) and the corresponding theoretical curves for the same four intensities (open circles).

After a reasonable choice of some critical parameters of the model (band tail parameter for electrons to be $\alpha=1$ and for holes to be $\alpha=0.5$, and attempt-to-escape frequency $v=5 \times 10^{19} \mathrm{~s}^{-1}$ ) [19], one can then combine $\mu_{n}^{0} \tau_{n}^{R}$ obtained from SSPC and $\tau_{n}^{\mathrm{R}}$ on undoped (or slightly n-type) samples to get a value of the electron band mobility. Using this procedure on a series of samples deposited at substrate temperatures $\mathrm{T}_{\mathrm{s}}$ between 100 and $320^{\circ} \mathrm{C}$, we were able to demonstrate an increase of the band mobility $\mu_{n}^{\circ}$ with an increase of $\mathrm{T}_{\mathrm{s}}$ up to $200^{\circ} \mathrm{C}$ (see Fig. 11). With the exception of the value for the sample deposited at $100^{\circ} \mathrm{C}$, mobilities $\mu_{n}^{\circ}$ obtained for this series agree quite well with other published data found in the literature (1.5-6 by Juska et al. [20], around 7 by Devlen et al. [21], 7-26 by Spear et al. [22]). The observe variation in $\mu_{n}^{0}$ with $T_{s}$ follows, furthermore, the same trend as the microstructure parameter $R$ for VHF-GD grown material $[23,24]$. $R$ is given here as the hydrogen content in the film in $\mathrm{SiH}_{2}$ or $\mathrm{SiH}_{3}$ bonding configurations divided by the total hydrogen content, both measured by IR spectroscopy [25]. A strong increase in $\mathrm{R}$ is generally considered as an indicator for the material becoming more porous.

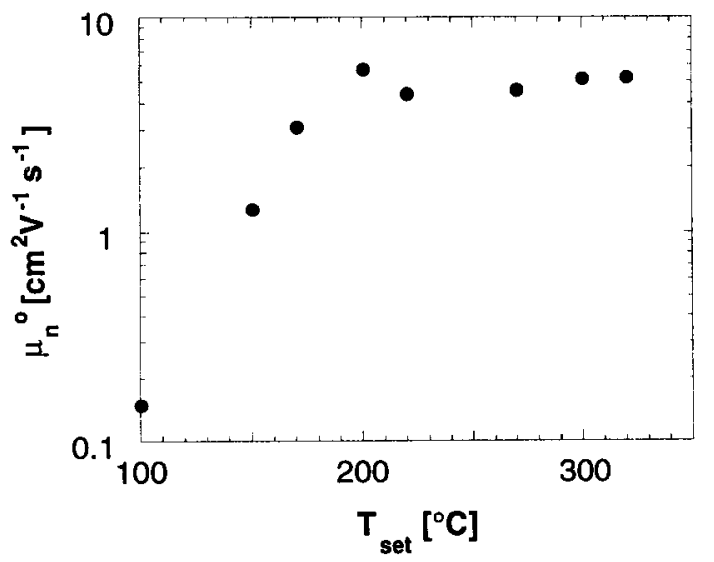

Fig. 11: Variation in the electron band mobility for a series of films grown at various deposition temperatures, for the selected (see text) choice of parameter. 


\section{Conclusions}

Several aspects of a-Si:H transport, involving mobilities, lifetimes and mobility-lifetime products, have been presented and discussed within the framework of a simple recombination model via dangling bond states.

A solution of the so-called $\mu \tau$-problem has been presented (at least a "qualitative" solution); in contrast with previous studies, this solution explains the observed effects by taking into account the difference in dangling bond occupation between dark (thermal equilibrium) conditions (as used for TOF) and steady-state illumination (as in case for SSPC and SSPG).

Comparison between a-Si:H material grown under different conditions (and exhibiting a variation in the position of their Fermi levels), as well as optimisation of corresponding solar cells certainly calls for the definition of a "true" quality-related transport parameter for the material. For this purpose, we have introduced a product $\mu^{\circ} \tau^{0}$ which takes into account both the variation in mobility and the variation in defect density in the material; the product $\mu^{\circ} \tau^{\circ}$ is, further, not dependent on the occupation of the dangling bonds. Using this quality parameter, we have been able to show a clear correlation between film properties and corresponding solar cell performance.

Finally, we have been able, from transient photoconductivity experiments in the small signal regime, to deduce values for the electron band mobility. The latter were shown to decrease strongly for deposition temperature below $200^{\circ} \mathrm{C}$.

\section{Acknowledgements}

The authors acknowledge the help of S. Dubail for sample preparation. This work was supported by the Swiss Federal National Science Foundation under grants (FN-32598 and FN-39377) and by the Swiss Federal Renewable Energy Program under Mandate (EF-REN(93)032).

\section{References}

[1] T. Tiedje, A. Rose, Solid State Comm. 37 (1981) 48.

[2] H. Orenstein, M.A. Kastner, Phys. Rev. Lett. 46 (1981) 1421.

[3] J. Kocka et al., Phil. Mag.B 63 (1991) 221.

[4] J. Hubin et al., Phil. Mag. Lett. 66 (1992) 115.

[5] F. Vaillant et al., Phys. Rev. B34 (1986) 4088.

[6] A. Shah et al., J. of Non-Cryst. Sol. 164-166 (1993) 485.

[7] D. Ritter et al., J. Appl. Phys. 62 (1987) p. 4563.

[8] N. Beck et al., Proc. of the $11^{\text {th }}$ EC-Photovoltaic Solar Energy Conference (1992) p. 625.

[9] N. Beck et al., MRS Proc. 297 (1993) p. 479.

[10] L. Yang et al., MRS Proc. 149 (1989) p. 563.

[11] M. Beck et al., Proc. of the $1^{\text {st }}$ World Conf. on Photovoltaic Energy Conversion, Hawaii, 1994 , in print.

[12] K. Hattori et al., J. of Non-Cryst. Sol. 137\&138 (1991) 363.

[13] N. Wyrsch et al., J. of Non-Cryst. Sol. 137\&138 (1991) 431.

[14] H. Curtins et al., Plasma Chem. \& Plasma Proc. 7 (1987) 267.

[15] E.A. Schiff et al., Phil. Mag. Lett. 55 (1987) 87.

[16] H. Antoniadis et al., J. of Non-Cryst. Solids 137\&138 (1991) 435.

[17] R.A. Street, Hydrogenated Amorphous Silicon, Cambridge University Press, (1991).

[18] P. Pipoz et al., J. of Non-Cryst. Sol. 164-166 (1993) 525.

[19] M. Goerlitzer et al., Proc. of the MRS spring meeting, San Francisco, 1995, to be published.

[20] G. Juska et al., Sol. State Com., 75 (1990) 531.

[21] R. I. Devlen et al., J. Non-Crys. Sol. 114 (1986) 567.

[22] W. E. Spear, in Amorphous Silicon and related materials, vol. B, 1st ed. (World Scientific Publishing Co. Pte. Ltd, Singapore, 1989), p. 760.

[23] U. Kroll et al., J. Non-Crys. Sol. 164-166 (1993) 59.

[24] F. Finger et al., J of Appl. Phys. 71 (1992) 5665.

[25] E. Bhattacharya, A.H. Mahan, Appl. Phys. Lett. 52 (1988) 1587. 
Hydrogenated Amorphous Silicon

doi:10.4028/www.scientific.net/SSP.44-46

Recent Progress in the Interpretation of a-Si:H Transport Properties: Lifetimes, Mobilities and Mobility-Lifetime Products

doi:10.4028/www.scientific.net/SSP.44-46.525 